\title{
Biotechnological Approaches on Anticancer Activity of Flavonoids-Mini Review
}

\author{
Selamoglu $\mathbf{Z}^{*}$ \\ Department of Medical Biology, Nigde Ömer Halisdemir University, Turkey \\ *Corresponding author: Zeliha Selamoglu, Department of Medical Biology, Faculty of Medicine, Nigde Ömer Halisdemir University, Nigde, 51240 \\ Turkey \\ Submission: 非 November 07, 2017; Published: 拝 November 15, 2017
}

\section{Introduction}

The approach to cancer prevention is based on there cognition that human cancer has multifactorial etiology and evolves through several molecular and cellular events occurring over many years following exposure to carcinogens [1].

Cancer is a multifactorial disease, which affects people of all ages. Some therapies remain the most widely adopted approach against some various of cancer diseases. Recently, oncologists and other specialist doctors have turned their attention towards the use of dietary supplements in cases of patients undergoing chemotherapy, due to their fears that antioxidant agents can disturb the working mechanism of chemotherapeutic agents and in consequence reduce or increase their therapeutic efficiency [1,2]. Many in vitro and in vivo studies suggest that flavonoids have effect for the prevention and treatment of some health disorders. It has been showed that protective activity of rich foods with flavonoids reduced risk of developing cancer $[1,3,4]$.

Flavonoids represent a large and diverse group of phenolic compounds derived from higher plants. Flavonoids are potent bioactive compounds that have anticarcinogenic effects since they can interfere with the initiation, development and progression of cancer by the modulation of cellular proliferation, apoptosis and metastasis. Many of these compounds, such as plant phenolics, often exhibit antioxidant activities; therefore the addition of these compounds into food products may be helpful to health the of consumers and also to the stabilization of food products. Due to the presence of some of these effective compounds such as flavonoids, phenolic acids and their esters in natural products such as plants and their extracts, if the positive physiological properties and the non-toxicity of the these products are proven it could be used as a mild antioxidant and preservative [5-7].

In this regards, research interest has focused on some natural compounds that have antitumor activities that may be useful adjuncts in supporting reduce the risk of cancer. Morever, natural compounds an attractive issue for designing new therapeutic agents as many chemical diversity found in plants and other livigs. Epidemiological works have implied the protective effects of bioactive natural compounds such as flavonoids in plant products against a wide variety of cancers and their ability to reduce oxidative damage, enhance exert anticancer effects $[1,3,4]$.

\section{Conclusion}

Finally, biotechnology has been getting an increasing consideration towards cancer cure due to their distinctive biomedical tratments to overcome the targeted drug delivery, designed drug solubility and drug toxicity.

\section{References}

1. Kim KH, Moon E, Choi SU, Kim SY, Lee KR (2013) Polyphenols from the bark of Rhus verniciflua and their biological evaluation on antitumor and anti-inflammatory activities. Phytochemistry 92: 113-121.

2. Hosseini A, Ghorbani A (2015) Cancer therapy with phytochemicals: evidence from clinical studies. Avicenna J Phytomed 5(2): 84-97.

3. Biswal BM, Sulaiman SA, Ismail HC, Zakaria H, Musa KI (2013) Effect of Withania somnifera (Ashwagandha) on the development of chemotherapy-induced fatigue and quality of life in breast cancer patients. Integr Cancer Ther 12(4): 312-322.

4. Cui Y, Shu XO, Gao YT, Cai H, Tao MH, et al. (2006) Association of ginseng use with survival and quality of life among breast cancer patients. Am J Epidemiol 163(7): 645-653.

5. Selamoglu Z (2017) Polyphenolic Compounds in Human Health with Pharmacological Properties. J Tradit Med Clin Natur 6(4): e137.

6. Selamoglu Z, Ustuntas HE, Ozgen S (2016) Traditional and complementary alternative medicine practices of some aromatic plants in the human health. Res J Biol 4(2): 52-54.

7. Shahidi F, Ambigaipalan P (2015) Phenolics and polyphenolics in foods, beverages and spices: Antioxidant activity and health effects-A review. J FunctFoods 18(Part B): 820-897. 